\title{
The Adaptation of the Short Dark Triad Personality Measure - Psychometric Properties of a German Sample
}

\author{
Marta Malesza ${ }^{1,2}$ - Pawel Ostaszewski ${ }^{3}$ - Susanne Büchner ${ }^{4}$. \\ Magdalena Claudia Kaczmarek ${ }^{5}$
}

Published online: 11 August 2017

(C) The Author(s) 2017. This article is an open access publication

\begin{abstract}
This research was designed to adapt and investigate the psychometric properties of the Short Dark Triad measure (Jones and Paulhus Assessment, 21(1), 28-41, 2014) in a German sample within four studies (total $N=1463$ ); the measure evaluates three personality dimensions: narcissism, psychopathy, and Machiavellianism. The structure of the instrument was analysed by Confirmatory Factor Analyses procedure. It indicated that the three-factor structure had the best fit to the data. Next, the Short Dark Triad measure was evaluated in terms of construct, convergent and discriminant validity, internal consistency $(\geq .72)$, and test-retest reliability during a 4-week period $(\geq .73$ ). Concurrent validity of the SD3 was supported by relating its subscales to measures of the Big Five concept, aggression, and self-esteem. We concluded that the Short Dark Triad instrument presented high cross-language replicability. The use of this short inventory in the investigation of the Dark Triad personality model in the German language context is suggested.
\end{abstract}

Marta Malesza

marta.malesza@psych.uw.edu.pl

1 Department of Psychology, University of Potsdam, Potsdam, Germany

2 Faculty of Psychology, University of Warsaw, Warsaw, Poland

3 SWPS University of Social Sciences and Humanities, Warsaw, Poland

4 Max Planck Institute for Research on Collective Goods, Bonn, Germany

5 Department of Psychosocial Medicine and Psychotherapy, University Hospital Jena, Jena, Germany
Keywords Short dark triad · SD3 - Adaptation · Reliability · Validity

\section{Introduction}

The Dark Triad, comprising psychopathy, Machiavellianism, and narcissism, represents a set of socially aversive and subclinical maladaptive personality characteristics. Psychopathy is characterized by high thrill-seeking, callousness, interpersonal antagonism, manipulation, and anti-social behavioral style (Hare and Neumann 2008). Machiavellianism is defined by self-interest and tendencies toward deceptiveness, exploitation and manipulation of others, cynical perspective on life and interpersonal relationships (Christie and Geis 1970). Finally, individuals high in Narcissism tend to focus largely on themselves, are characterized by self-absorption, dominance, and feelings of entitlement and grandiosity, as well as devaluation of others (Emmons 1987). There has been an asymptotic rise in papers investigating the utility of these traits (for a review, see Furnham et al. 2013). Recent papers have found that the Dark Triad traits are differentially informative in predicting workplace behaviors, interpersonal behaviors, mating behaviors, antisocial behaviors, aggression, and financial misbehaviors (e.g., Jones and Paulhus 2010; Lee and Ashton 2005; Malesza and Ostaszewski 2016a, b).

\section{Measures of the Dark three Constructs}

Most studies used three separate assessment tools for each of the Dark Triad traits, typically the Self-Report Psychopathy Scale, the Machiavellian Scale, and the Narcissistic Personality Inventory (for a review, see Furnham et al. 2013). Consequently, traditional measures of the Dark Triad together require approximately 100 items to complete. If time 
is limited, using concise measures can eliminate item redundancy, save time and effort, and thus reduce participant fatigue (Gosling et al. 2003). To meet the need for a brief assessment method, two short measures of the Dark Triad were developed: Short Dark Triad (Jones and Paulhus 2014) and the Dirty Dozen (Jonason and Webster 2010). The first measure comprises 27 items, whereas the second instrument includes 12 items. However, the second tool - the Dirty Dozen - is controversial. Although some researchers have found it to be useful (Jonason and Luévano 2013; Rauthmann, 2012), the instrument has been critiqued in several recent reports (Jones and Paulhus 2014; Lee et al. 2013; Maples et al. 2014; Miller et al. 2012). The main limitation is the psychometric cost of using this short measure. Particularly, compared with standard long multi-item measures of the Dark Triad, the Dirty Dozen converges less strongly with other Dark Triad measures and shows weaker convergent validity with other external variables (e.g., the Big Five, aggression, and self-esteem level; Maples et al. 2014; Miller et al. 2012).

\section{Short Dark Triad Measure}

A brief proxy measure - the Short Dark Triad questionnaire (SD3; Jones and Paulhus 2014) - is a self-report questionnaire developed to assess three dimensions of the Dark Triad personality model. First, to develop the SD3 measure, after revision of the seminal literature, Jones and Paulhus (2014) created a large item pool that was subjected to further analyses. Next, the authors reduced the number of items as much as possible without missing the conceptual facets of each triad variable. The result was a 27 -item instrument. Using the Principal Components Analysis, after studying several rotated structures ranging from three to six factors, three composites, consistent with the formulation of the Dark Triad model, were formed. Moreover, previously reported psychometric properties of the original SD3 revealed acceptable internal consistency for every dimension and convergent validity with the external variables consistent with hypotheses (Furnham et al. 2013; Jones and Paulhus 2014; Lee and Ashton 2005; Paulhus and Williams 2002). Jones and Paulhus (2014) concluded that “...the SD3 subscales provide useful proxies for the established Dark Triad measures they were meant to replace" (p. 37).

\section{Overview of the Present Study}

In the present report, we aimed to adapt the SD3 inventory in a sample of German adults. The second purpose of this study was to determine whether the original structure of the SD3 measure described in terms of the three-factor model by Jones and Paulhus (2014) could be replicated with a German version by Confirmatory Factor Analyses and to examine the psychometric properties of the adaptation of this instrument.
Thus, our first task focused on the translation process of the original items from the measure. The process is precisely described. Next, analyses of the measure structure through Confirmatory Factor Analyses with Maximum Likelihood estimation method were performed. We examined the reliability of the SD3 proxy. We continued with examining if the measure possesses acceptable construct validity by comparing it with longer established measures of the Dark Triad. Finally, we examined patterns of convergent and discriminant relationships with other relevant variables of personality that have been proven important in prior research, specifically the Big Five, self-esteem and aggression levels. Developing the same measure for different countries has the advantage of establishing the same metric for all countries analysed (Furr \& Bacharack, 2008). This characteristic would largely facilitate cross-cultural research with the Dark Triad personality model. With that said, if a particular measure retains sound psychometric characteristics when used in different cultures, then that measure has several things to recommend it (Hambleton 2001). First, it would provide a single measure that was potentially useful to researchers and practitioners in multiple cultures. Second, such a research should confirm its psychometric characteristics when used outside of the culture in which it was originally developed.

\section{Study 1: Factor Structure of the Adapted Short Dark Triad Measure}

The aim of the first study was to provide information about the factor structure of the adapted SD3 measure. For the factorial structure, a 3-factor structure, coherent with the model and the factor analysis of the original version, was found (Jones and Paulhus 2014; Paulhus and Williams 2002) (i.e., each dimension loads specifically on its respective dimensions, with intercorrelated dimensions).

Participants The first sample was collected from the internet using an online version of the SD3 measure. The sample was composed of 598 adults ( 347 female and 251 male) of all ages ranging from 18 to 67 years $(\mathrm{M}=27.34, \mathrm{SD}=7.50)$, and approximately $40 \%$ of them were aged between 19 and 27 . The majority of the participants had a higher education: 327 were university graduates, and 195 had a high school education, whereas 76 had a lower level of education. 178 participants were students at the time of data collection. The nonstudent respondents were from various professions.

Procedure Participation was voluntary and anonymous. Subjects were not compensated in any way. Data were collected in an online study administered via a tool for online surveys: SosciSurvey facility. Individuals were recruited through advertisements posted on social media (i.e., Facebook). All 
participants provided a consent which was obtained online after a detailed instruction describing main purposes and approximate duration of the study. All studies were approved by the appropriate ethics review committee prior to initiation. All individuals were offered feedback on general results of the study.

Measure Subjects completed the translated version of the SD3 measure, which consisted of 27 items (sample item: It's not wise to tell your secrets). It was the only instrument which participants were asked to complete. As in the original version, responses were given on a 5-point Likert scale from 1 (strongly disagree) to 5 (strongly agree). Before the individuals could send their answers over the net, the data were checked for completeness.

\section{Translation}

To ensure that the items resemble the meaning of the original English items as closely as possible, we followed a common procedure of back-translation in which a text is translated from a source into a target language, and a second interpreter independently translates the text back into the source language. Translation and back-translation of the original inventory and the new scale were provided by professional interpreters. The English version of the measure was first translated into German and then back-translated into English by two translators, according to the guidelines developed by the International Test Commission (Hambleton 2001). Afterwards, both translators compared the original version and the back-translated version for equivalence of meaning. The accuracy of the translation was evaluated by comparing the original and back-translated versions. Any discrepancy was discussed until an agreement was reached. This version was then refined by paying special attention to the use of frequently-used words, and using a correct and easy grammar in order to ensure the items were well understood for every level of education.

Before examining its psychometric properties, the German version of the SD3 was submitted to a pretest to ensure comprehensibility and clarity of items, as well as to make sure that items were worded in an understandable language for the German-speaking population (Hambleton 2001). Criteria were judged by a group of sixty-five adult individuals ranging in age from 20 to 48 years. The sample consisted of students (60\%) and employees of various professions. The mean age was 27.2 years $(\mathrm{SD}=3.05)$, and $73 \%$ were female. They were asked to rate each item on its clarity using a 7-point Likert scale from 1 (not clear at all) to 7 (perfectly clear) and also to circle every item or part of item that they did not understand. Using a 7-point rating scale and following Hambleton's (2001) suggestions, items with a score 5 or below were reviewed. Based on these item-metric analyses, three items were corrected by paying special attention to the use of frequent and well-known words. In item 'There are things you need to keep hidden from others in order to save face', the translators evaluated a change in the expression 'to save face' for the expression 'um dein Ansehen zu wahren' (protect your reputation), thus reducing the possibility of error in interpretation. Next, in item 'I know I am special because everyone keeps telling me', the expression 'ständig' (constantly) was added, with the aim of emphasizing the occurrence. Finally, in item 'You must get important people on your side, no matter the cost', the judges evaluated a change in the adjective 'important' for the adjective 'wichtigen' (essential). Finally, this last version was given to the University Language Advisor for final correction. The German translation of the SD3 is displayed in Appendix.

Statistical Analyses To examine the factor structure of the German SD3, we performed the Confirmatory Factor Analyses with the AMOS statistical package (Arbuckle 1997). The estimation method was Maximum Likelihood (ML). Note that, ML makes assumption about multivariate normality. As both skewness and kurtosis were less than or equal to 1.3 for all items, indicating that the item-distributions were similar to the rest of the items in the instrument and that the item distributions were rather symmetrical, the ML estimation was considered appropriate (see Mulaik 2007). We used several criteria of model fit (see Bollen and Long 1993). A well-fitting model should ideally have a nonsignificant $\chi^{2}$ statistic $(p>.05)$. The model is considered to have a good fit if the GFI (goodness-of-fit index), TLI (Tucker-Lewis index), and CFI (comparative fit index) values are approximately .95. As the $\chi^{2}$ statistic tends to be inflated in small samples, the ratio $\chi^{2} / \mathrm{df}$ was determined, which should not be much larger than 2.0. The $\chi^{2} / d f$ is a measure of the absolute fit of the model with the data, indicating how closely the model fits compared to a perfect fit. The RMSEA (root mean square error of approximation) represents reasonable errors of approximation in the population; a value of approximately .05 or less would indicate a close fit and a value of up to .08 would represent a reasonable fit of the model. We note, however, that the choice of indices and cutoff values is a topic surrounded by considerable controversy (see, e.g., Mulaik 2007). In these analyses, two models were examined: (1) one-factor solution (all 27 items were combined into one factor); and (2) three-factor solution, which comprised three factors representing three Dark Triad traits. The choice of models was influenced by Jones and Paulhus (2014). Next, to evaluate the internal consistency, Cronbach's $\alpha$ of scales were calculated. SD3 scale inter-correlations were checked with two-tailed Pearson correlations.

Results and Discussion The three-factor model had a good fit to the data from German sample: $\chi^{2}(102 \mathrm{df})=206.24, p>.05$; $\chi^{2} / \mathrm{df}=2.02 ;$ RMSEA $=.04, \mathrm{GFI}=.97, \mathrm{CFI}=.98, \mathrm{TLI}=.99$. 
Second, for the purpose of comparison, a one-factor model, which presupposes that all the items pertain to the same factor, was also assessed. According to the $\chi^{2}$ statistic and the $p$ value, the model would have to be rejected $\left(\chi^{2}[114\right.$ $\mathrm{df}]=485.20, p<.01 ; \mathrm{RMSEA}=.08, \mathrm{GFI}=.83, \mathrm{CFI}=.89$, $\mathrm{TLI}=.88)$. The value $\chi^{2} / \mathrm{df}=4.26$ obtained here also indicates an unacceptable fit. The three-factor model had a much better fit than a one-factor model of general Dark Triad, $\Delta \chi_{(12)}^{2}=278.96, p<.01$.

Based on the standardized regression weights, each item was linked to a single factor. All of these coefficients exceed .45 , providing additional support for the efficacy of this model (see Table 1). Also, the corrected item total correlation was positive on all items, ranging from .47 to .91 . This indicates that all contribute to gauge what the test measures and further in the same direction. All items were encapsulated in their respective factors as in the original distribution of the instrument (see Jones and Paulhus 2014).

Moreover, the following moderate correlations between the three SD3 composite scales were found for the examined sample: $r_{\text {Mach.Narc }}=.34, \mathrm{r}_{\text {Mach.Psych }}=.48$ and $\mathrm{r}_{\text {Narc.Psych }}=.41$ (all $p<.01$ ). These results are comparable with the correlations reported by Jones and Paulhus (2014). As shown in the descriptions of the three dark traits (Paulhus and Williams 2002), there are several notable common features among these variables. All of them include the core of manipulation and exploitation (Lee et al. 2013). Thus, it can describe the significant modest correlations between the Dark Triad factors.

The internal consistencies were in a medium but acceptable range (narcissism $\alpha=.73$, psychopathy $\alpha=.72$, Machiavellianism $\alpha=.78$ ). Each dark factor is a basic personality trait that covers a wide range of behavioral and emotional reactions. Multi-item scales can afford to bolster internal consistency by using several items with high content overlap (Gosling et al. 2003). In contrast, with only nine items per scale, the SD3 instead emphasized content validity considerations, resulting in lower Cronbach's alpha. Thus, researchers wishing to correct the SD3 correlations for unreliability should base their corrections on reliability estimates that are less biased by the small number of items on each scale. Recent work (Crede et al. 2012) suggests that test-retest reliability may be more predictive than estimates of internal consistency.

\section{Study 2: Test-Retest Reliability}

Consistency over time is a defining feature of personality traits (Furr \& Bacharack, 2008), and therefore, such evidence will bolster the claims about the usefulness of our measure as well as provide support for the treatment of the Dark Triad as stable personality variables in an adapted instrument. Here, the testretest reliability of the SD3 measure was assessed during a 4week period.
Table 1 Standardized regression weights of the adapted Short Dark Triad items

\begin{tabular}{|c|c|c|c|}
\hline \multirow[t]{2}{*}{ No. of item } & \multicolumn{3}{|c|}{ Study $1(N=598)$} \\
\hline & $\mathrm{F} 1$ & $\mathrm{~F} 2$ & F3 \\
\hline 1. & .75 & & \\
\hline 2. & .68 & & \\
\hline 3. & .67 & & \\
\hline 4. & .45 & & \\
\hline 5. & .67 & & \\
\hline 6. & .57 & & \\
\hline 7. & .77 & & \\
\hline 8. & .95 & & \\
\hline 9. & .48 & & \\
\hline 10. & & .46 & \\
\hline $11 .^{\mathrm{R}}$ & & -.55 & \\
\hline 12. & & .53 & \\
\hline 13. & & .67 & \\
\hline 14. & & .69 & \\
\hline $15^{\mathrm{R}}$ & & -.71 & \\
\hline 16. & & .49 & \\
\hline $17 .^{\mathrm{R}}$ & & -.58 & \\
\hline 18. & & .73 & \\
\hline 19. & & & .53 \\
\hline $20 .^{\mathrm{R}}$ & & & -.55 \\
\hline 21. & & & .54 \\
\hline 22. & & & .57 \\
\hline 23. & & & .47 \\
\hline 24. & & & .49 \\
\hline $25^{\mathrm{R}}$ & & & -.67 \\
\hline 26. & & & .64 \\
\hline 27. & & & .62 \\
\hline
\end{tabular}

The exact meaning of 27 items can be found in "Introducing the Short Dark Triad (SD3): A brief measure of dark personality trait" by D.N. Jones and D.L. Paulhus, 2014, Assessment, 21(1), p. 38 (Appendix)

$R$ reverse-scored items; F1 = Machiavellianism scale; F2 = Narcissism scale; F3 = Psychopathy scale

Participants To evaluate SD3 test-retest stability, for 221 subjects of the 598 participants who completed the SD3 measure in Study 1, we obtained a second evaluation of three dark traits; individuals completed the SD3 again 4 weeks after the first administration. Volunteers $(N=221 ; 66 \%$ female; mean age 22.3 years; $\mathrm{SD}=2.3$ ) from unique IP addresses recruited via the Internet completed an online survey that informed them of the nature of the study and asked them to respond to the items described above. Again, subjects were not compensated in any way. All respondents taking part in the research provided informed consent after the nature of the study had been explained to them. All individuals were offered feedback on general results of the study after the second measurement. They did not get any feedback after Study 1 until Study 2 was completed. 
Measures Study 2 used the same 27-item SD3 measure used previously in Study 1.

Results and Discussion Test-retest reliability of the SD3 measure and its three components was determined by correlating Session 1 with Session 2 scores (as well as their 95\% confidence intervals). The test-retest values for the various scales were as follows: Machiavellianism $\mathrm{r}_{\mathrm{tt}}=.81(95 \% \mathrm{CI}=.80-.82 ; 179$ individuals out of 221 demonstrated stable preferences), psychopathy $\mathrm{r}_{\mathrm{tt}}=.83(95 \% \mathrm{CI}=.81-.85 ; 183$ individuals out of 221 demonstrated stable preferences), and narcissism $\mathrm{r}_{\mathrm{tt}}=.74$ (95\% CI $=.73-.75 ; 164$ individuals out of 221 demonstrated stable preferences). These correlations were uniformly high and indicate good test-retest stability of the SD3 measure.

\section{Study 3: Construct Validation against Standard Measures}

A crucial aspect of validating an adapted assessment method is a study that tests its performance against the standard assessment tools (Crede et al. 2012). Specifically, to what degree and in what way do the SD3 subscales correlate with the long-form measures of the Dark Triad. Accordingly, Study 3 included the standard Dark Triad measures-Mach IV, SRP-III, and NPI - in addition to the SD3 tool. For comparison, an alternative short proxy - the 12-item Dirty Dozen (DD) measure - was included. To assess construct validity, some basic predictions were made. The SD3 measure should behave similarly to the longer measures. Thus, the SD3 should be correlated with the longer, standard measures of the Dark Triad. Moreover, because multiitem inventories tend to be more reliable than singleitem inventories (Gosling et al. 2003), correlations between the SD3 measure and long instruments of the Dark Triad should be stronger than between DD and the same three longer measures.

Participants A total of 384 German students participated in the study (154 men and 230 women, ranging in age from 20 to 26 years, $\mathrm{M}=23, \mathrm{SD}=1.8$ ). Individuals were recruited through advertisements posted around the university campus. All subjects provided informed consent or assent after the nature of the study had been explained to them. Participants were not compensated in any way. All individuals were offered feedback on general results of the study.

Measures The adapted Short Dark Triad 27-item scale was used. An alternative short assessment method of the Dark Triad, the Dirty Dozen measure (Küfner et al. 2014) comprising 12 items, was used (sample item: I tend to lack remorse). Responses are scored on a 9-point Likert scale ranging from 1 (strongly disagree) to 9 (strongly agree). Additionally, the standard long Dark Triad measures were provided. Machiavellianism was assessed with an 18-item scale (sample item: The best way to handle people is to tell them what they want to hear; Henning and Six 2008). The answer format was a six-point Likert-type scale from 0 (not at all like me) to 5 (totally like me). Psychopathy was measured with a 30-item version of the Self-Report Psychopathy Scale-III (sample item: I can be fairly cunning if I have to be; Williams et al. 2003). Answers were given on a five-point Likert-type scale from 0 (not at all like me) to 4 (totally like me). Narcissism was assessed with the 17-item Narcissistic Personality Inventory (sample item: I am essentially a modest person; von Collani 2008) with a five-point Likert-type scale from 0 (not at all like $m e$ ) to 4 (totally like me). The standard Dark Triad measures were divided into their respective facets to determine if the SD3 subscales were capturing each measure in a balanced way (see Jones and Paulhus 2014).

Procedure All measures were paper-and-pencil questionnaires and were presented in a random order.

Results and Discussion The relationships between the SD3 scales in relation to established long Dark Triad measures and an alternative DD tool were examined (see Table 2). In sum, all the SD3 scales showed a clear correspondence with their criterion counterparts measured by the three longer tools. Moreover, compared with the Dirty Dozen versions, all three Short Dark Triad subscales showed stronger correlations with the facets of its corresponding standard, ranging from .28 (Antisocial Behavior facet of Psychopathy Scale with the SD3 Narcissism) to .71. (Manipulation facet of Psychopathy Scale with the SD3 Psychopathy).

These results add support for previous research suggesting that scores from the SD3 scales manifested acceptable convergent validity with longer, more established measures (Jones and Paulhus 2014; Maples et al. 2014; Miller et al. 2012). The SD3 adequately covers the elements of each Dark Triad construct. Additionally, direct comparisons with the DD instrument indicated stronger construct validity with the wellestablished longer tools of the SD3 than of the DD measure. These results confirm previous findings showing that the SD3 scales provide a more complete account of the underlying Dark Triad constructs (Maples et al. 2014). Notably, the DD psychopathy and narcissism scales seemed to have only scattered coverage of the longer Dark Triad measures. The stronger convergent validity with the established Dark Triad measures of the SD3 scores adds support for the claim that only 12 items of the DD measure are likely to contain only a particularly narrow aspect of the Dark Triad constructs (Maples et al. 2014). 
Table 2 Convergent correlations between traditional three measures of the Dark Triad and the Short Dark Triad and Dirty Dozen measures (top panel), and correlations among the Short Dark Triad and Big-Five, aggression, and self-esteem measures (lower panel)

\begin{tabular}{|c|c|c|c|c|c|c|}
\hline \multirow[b]{3}{*}{ SRP facets } & \multicolumn{2}{|c|}{ Psychopathy } & \multicolumn{2}{|c|}{ Narcissism } & \multicolumn{2}{|c|}{ Machiavellianism } \\
\hline & \multicolumn{6}{|c|}{ Study $3(N=384)$} \\
\hline & SD3 & $\mathrm{DD}$ & SD3 & DD & SD3 & DD \\
\hline Manipulation & $.71^{* *}$ & $.42 * *$ & $.41 * *$ & $.29 *$ & $.55 * *$ & $.40^{* *}$ \\
\hline Callous Affect & $.43^{* *}$ & .06 & $.30 * *$ & $.18 *$ & $.49 * *$ & $.60^{* *}$ \\
\hline Erratic Lifestyle & $.52^{* *}$ & .01 & $.45^{* *}$ & .07 & $.43^{* *}$ & $.33^{* *}$ \\
\hline Antisocial Behavior & $.51^{* *}$ & $.37 * *$ & $.28^{*}$ & $.39^{* *}$ & $.29 *$ & $.46^{* *}$ \\
\hline \multicolumn{7}{|l|}{ NPI facets } \\
\hline Leadership/Authority & $.44^{* *}$ & $.31 * *$ & $.50 * *$ & $.37^{* *}$ & $.32 * *$ & $.29 *$ \\
\hline Exploitative/Entitlement & $.37^{* *}$ & $.29 * *$ & $.49 * *$ & $.55^{* *}$ & $.41 * *$ & $.26^{*}$ \\
\hline \multicolumn{7}{|c|}{ Mach-IV facets } \\
\hline Machiavellian Tactics & $.42^{* *}$ & $.30 * *$ & $.31 * *$ & $.19^{*}$ & $.53 * *$ & $.43^{* *}$ \\
\hline Cynical Worldview & $.49^{* *}$ & $.46^{* *}$ & .09 & $.17^{*}$ & $.65^{* *}$ & $.58 * *$ \\
\hline & \multicolumn{6}{|c|}{ Study $4(N=481)$} \\
\hline \multicolumn{7}{|c|}{ Big Five personality traits } \\
\hline Agreeableness & $-.40 * *$ & & $-.25 * *$ & & $-.27 * *$ & \\
\hline Conscientiousness & $-.21 *$ & & $.22 *$ & & $-18^{*}$ & \\
\hline Emotional Stability & -.01 & & -.08 & & .00 & \\
\hline Extraversion & -.04 & & .09 & & -.02 & \\
\hline Intellect/Imagination & .00 & & .01 & & -.01 & \\
\hline \multicolumn{7}{|c|}{ Aggression Questionnaire } \\
\hline Physical Aggression & $.39^{* *}$ & & -.02 & & $.24 * *$ & \\
\hline Verbal Aggression & $.40^{* *}$ & & .01 & & $.43^{* *}$ & \\
\hline Anger & $.24^{* *}$ & & .00 & & .02 & \\
\hline Hostility & -.04 & & $.16^{*}$ & & $.29 * *$ & \\
\hline Self-esteem & .07 & & .06 & & -.01 & \\
\hline
\end{tabular}

$D D$ Dirty Dozen, SD3 Short Dark Triad, SRP-III Self-Report Psychopathy Scale-III, Mach-IV Machiavellianism scale, NPI Narcissistic Personality Inventory

$* p<.05 ; * * p<.01$

\section{Study 4: Convergent and Discriminant Validity}

The aim of Study 4 was to provide additional evidence of the SD3 measure's psychometric properties and its validity as a concise measure of constructs underlying the Dark Triad. It is common to use the external personality traits to describe the validity of measures (Gosling et al. 2003). The validity of the SD3 measure was assessed by correlating it with the Big Five measure, aggression questionnaire, and a global measure of self-esteem-constructs that have proven important in prior studies with the original, 91-item version of the Dark Triad assessment technique (for a review, see Furnham et al. 2013). The original SD3 measure already has been successfully used in many research contexts (for a review, see Furnham et al. 2013), providing insight into darker personality factors and suggesting that the Dark Triad model may be theoretically informative compared to other models. The conceptual formulation of the Dark Triad traits is that they share disagreeableness (Furnham et al. 2013; Jonason et al. 2009; Lee and Ashton 2005; Paulhus and Williams 2002), conscientiousness (Furnham et al. 2013; Lee and Ashton 2005), and aggression (e.g., Jones and Paulhus 2010; Bushman and Baumeister 1998), which may relate to a fast life strategy that underlies the nature of the Dark Triad (e.g., Jonason et al. 2010). The SD3 measure should be correlated negatively with agreeableness and conscientiousness from the five-factor model of the Big Five and positively with aggression. In contrast, in showing evidence of discriminant validity, it was predicted that the SD3 should be mostly uncorrelated with the measure of selfesteem level (Bushman and Baumeister 1998). Last, there is ample evidence that suggests that men score higher on all three of these traits than women (e.g., Furnham et al. 2013; Jonason et al. 2009), and therefore, we expect that men should score higher than women on the SD3 measures. These gender predictions constitute validity tests of the SD3 measure (Furr and Bacharach 2008).

Participants The sample consisted of 481 participants (270 women and $211 \mathrm{men}$ ).

Participants were students from all levels ( $72 \%$ of the sample) and employees of various occupations (ages between 18 and 61 years; $M=28 ; S D=3.1$ ). Subjects were recruited through advertisements posted on social media (i.e., Facebook). All respondents taking part in the research provided informed consent after the nature of the study had been explained to them. They were not compensated in any way. All individuals were offered feedback on general results of the study.

Materials Study 4 used the same 27-item Short Dark Triad instrument used previously in three studies. Participants also completed a battery of other German-speaking measures. Participants responded to a 50-item Big Five personality dimension measure based on the International Personality Item Pool (sample item: Don't like to draw attention to myself; Goldberg et al. 2006). The instrument contains five factors (Extraversion, Agreeableness, Conscientiousness, Emotional Stability, and Intellect/Imagination (mean $\alpha=.84$ )). Aggression was measured using a 29-item version of the Aggression Questionnaire (sample item: I let my anger show when I do not get what I want; von Collani and Werner 2005) with 29 items with the four components of Physical Aggression, Verbal Aggression, Anger, and Hostility (mean $\alpha=.79$ ). Finally, the global self-esteem level was assessed with a 10-item measure of self-esteem (sample item: At times I think I am no good at all; von Collani and Herzberg 2003; $\alpha=.69$ ). All measures used a 5 -point Likert response scale ranging from 1 (strongly disagree) to 5 (strongly agree).

Procedure The study was conducted using the paper-andpencil method. All questionnaires were presented in a random order.

Results and Discussion We attempted to replicate the factor structure of the SD3 through the use of CFA as in Study 1. The 
fit indices were as follows: $\chi^{2}(64 \mathrm{df})=138.17, p>.05 ; \chi^{2} /$ $\mathrm{df}=2.16 ;$ RMSEA $=.07 ; \mathrm{GFI}=.98, \mathrm{CFI}=.97, \mathrm{TLI}=.96$. The outcome of the CFA suggested that a three-factor solution yielded the best model fit. As with the data from Study 1, CFI, GFI, TLI, RMSEA, and $\chi^{2} / \mathrm{df}$, respectively, indicated a good or an acceptable model fit.

Correlations were used to explore convergent and discriminant validity between the SD3 measures and the Big Five, aggression, and self-esteem assessment tools. As expected, the SD3 displayed patterns of correlations that closely matched those obtained with the standard long Dark Triad instruments in previous research (Jones and Paulhus 2014; Maples et al. 2014; data from the present study are summarized in the lower part of Table 2). This correspondence adds further construct validity to the SD3 measure based on its location in personality space (Gosling et al. 2003).

Big five The SD3 measure and each scale were negatively associated with agreeableness. Individuals who are disagreeable in nature manifest characteristics that are antisocial, which is how these personality traits relate to the Dark Triad traits (Paulhus and Williams 2002). Additionally, the SD3 showed a significant association with conscientiousness. However, a difference emerged with the facets of SD3; the positive correlation with narcissism was due to achievementstriving and competence, whereas the negative correlations with Machiavellianism and psychopathy were caused by low dutifulness and low deliberation (Lee and Ashton 2005; Miller et al. 2012). Except for agreeableness and conscientiousness, none of the other three factors operationalized within the Big Five model correlated substantially with the three Dark Triad variables. However, in previous research narcissism significantly correlated with Intellect/Imagination and Extraversion (Lee and Ashton 2005), and in the present study it did not. As noted in previous research, Narcissism includes some traits (i.e., leadership, assertiveness, dominance, and a tendency to seek social attention) that are not the core elements within psychopathy and Machiavellianism, all of which are relevant to extraversion and intellect/imagination (Lee and Ashton 2005). However, this finding could be an issue of culture or idiosyncratic to the sample involved in the study as well.

Aggression The SD3 showed a consistent pattern of convergent validity with measures of aggression, suggesting that people who obtain high scores on the Dark Triad may also use aggression to obtain what they want (Jonason and Webster 2010). Among the SD3 scales, Machiavellianism was positively related to physical aggression, verbal aggression, and hostility. Psychopathy, in contrast, was positively related to anger, physical and verbal aggression. Narcissism, however, was positively related only to hostility. Those high in narcissism are easy to provoke into aggression but only if the provocation consists of a significant ego-threat (Bushman and
Baumeister 1998; Furnaham et al., 2013; Jones and Paulhus 2010). Thus, it can only describe the small relationship between narcissism and hostility.

Self-Esteem Study 4 also showed the significant lack of relationship between self-esteem stability and the Dark Triad traits (for contrary results for narcissism, see Bushman and Baumeister 1998).

Sex Differences Gender differences were examined using a ttest. After including a Bonferroni correction for multiple comparisons (the Bonferroni correction sets the significance cutoff at adjusted alpha level/number of t-tests performed. Thus, in the present data, with 3 tests and $\alpha=.05$, we would only reject a null hypothesis if the $p$-value is less than .016), tests for sex differences revealed men scored slightly higher than women on the scales of narcissism, $t(479)=3.90, p=.015$; and psychopathy, $t(479)=9.05, p<.01$; as well as the entire SD3 measure, $t(479)=14.17, p<.01$. The present study replicated prior findings indicating that high scores on these variables are more prevalent among men (Furnham et al. 2013). The gender differences in the Dark Triad traits can be described by the evolutionary perspective, in which men are thought to benefit more from social exploitation (Jonason et al. 2009). Thus, consequently, men gain higher scores on personality traits related to social exploitation.

\section{General Discussion}

The purpose of the present study was to provide a welladapted version of the Short Dark Triad measure based on the Dark Triad personality model proposed by Paulhus and Williams (2002). The additional aim was to determine whether the original structure of the SD3 measure described in terms of the three-factor model by Jones and Paulhus (2014) could be replicated with a German version. The measure displayed a robust structure and acceptable psychometric properties in the German language. Specifically, the aims of the present study were: to analyse the factor structure, internal consistency, and temporal stability of the SD3; to explore the association of the standard measure methods of the Dark Triad with the adapted SD3 inventory; and to investigate the convergent and discriminant validity of the SD3 measure.

CFA was used to evaluate if the previously reported factor structure of the SD3 was valid for our data. We used both model fit measures and $\chi^{2}$-difference scores in these model comparisons. Consistent with Jones and Paulhus (2014), the three-factor solution gave the best model fit. These factors were closely associated with the theoretical three dimensions (Paulhus and Williams 
2002). This solution was also supported by the $x^{2}$-difference test comparing three-factor model to one-factor model. Note that the RMSEA, GFI, and $\chi^{2} / \mathrm{df}$ all indicated a good or acceptable model fit. Internal consistency coefficients were acceptable and comparable with those reported by Jones and Paulhus (2014). The lower internal consistencies results could be due to the threefold fewer number of items compared to longer wellestablished Dark Triad instruments (27 versus 91 items). Moreover, in the present study, test-retest reliability of the adapted SD3 measure remains adequate in intervals of four weeks, indicating dark personality variables to be relatively stable over time traits. Additionally, results report an acceptable convergence among the SD3 and the other well-established Dark Triad instruments, confirming the outcomes found by Jones and Paulhus (2014).

Concurrent validity of the SD3 was assessed by relating its subscales to measures of the Big Five concept, aggression, and self-esteem. The analyses of convergent validity support both the validity of the German version of the SD3 as well as Paulhus' and Williams' (2002) concepts of basic personality traits. Consistent with the theoretical predictions, the Dark Triad traits are mainly related to a lack of agreeableness and to conscientiousness and to a stronger degree of various types of aggression, lending support to the validity of the adapted instrument. However, our research had some limitations.

\section{Limitations}

It is evident that our samples are also severely biased. There were very few participants from groups with lower education. Additionally, the retest reliability is high, but the period of time between the first and second assessments was short (four weeks). Finally, the validation of the adapted SD3 measure was done exclusively against other self-report instruments. In establishing the validity of a psychological construct, it clearly is important to consider non-self-report data as well (Furr and Bacharach 2008). Specifically, with self-report tasks, asking for reporting participants' behavioral tendencies in various contexts, may not always accurately reflect individuals' behavior. Self-reported measures are more sensitive to bias and are influenced by social desirability responses.

\section{Conclusions}

Despite the frequently cited evidence that the short measures of personality are likely to have several psychometric weaknesses than scores on well-constructed longer inventories (Crede et al. 2012), the results presented in this article suggest that these beliefs are generally incorrect. Overall, the present findings suggest that the German version of the SD3 instrument can stand as a reasonable proxy for a longer Dark Triad instrument, especially if research conditions dictate a short measure to be used.

Funding This research was supported, in part, by a scholarship from the German Research Foundation (Deutsche Forschungsgemeinschaft).

\section{Compliance with Ethical Standards}

Conflict of Interest Author Marta Malesza declares that she has no conflict of interest. Author Paweł Ostaszewski declares that he has no conflict of interest. Author Susanne Büchner declares that she has no conflict of interest. Author Magdalena Claudia Kaczmarek declares that she has no conflict of interest.

Ethical Approval All procedures performed in studies involving human participants were in accordance with the ethical standards of the institutional and national research committee and with the 1964 Helsinki declaration and its later amendments or comparable ethical standards.

Informed Consent Informed consent was obtained from all individual participants included in all studies.

\section{Appendix}

\section{Machiavellismus}

1. Es ist nicht klug seine Geheimnisse zu verraten.

2. Um mich durchzusetzen, neige ich dazu geschickte Manipulationen zu benutzen.

3. Was immer es auch kostet, du musst die wichtigen Personen auf deine Seite bringen.

4. Vermeide direkten Streit mit anderen, da sie in der Zukunft hilfreich für dich sein könnten.

5. Es ist klug, Informationen nachzugehen, die du später gegen Personen einsetzten kannst.

6. Um es jemandem Heimzuzahlen solltest du auf den richtigen Zeitpunkt warten.

7. Es gibt Dinge, die du vor anderen Menschen verbergen solltest, um dein Ansehen zu wahren.

8. Stell sicher, dass deine Pläne dir und nicht anderen nützen.

9. Die meisten Menschen können manipuliert werden.

Narzissmus

1. Menschen sehen in mir den geborenen Anführer.

2. Ich hasse es im Mittelpunkt der Aufmerksamkeit zu stehen.

3. Viele Gruppenaktivitäten neigen ohne mich dazu, langweilig zu sein.

4. Ich weiß, dass ich besonders bin, da es mir alle ständig sagen. 
5. Ich mag es mit wichtigen Leuten bekannt gemacht zu werden.

6. Ich fühle mich verlegen, wenn mir jemand ein Kompliment macht.

7. Ich wurde schon mit berühmten Personen verglichen.

8. Ich bin ein durchschnittlicher Mensch.

9. Ich beharre darauf, den Respekt zu bekommen, den ich verdiene.

\section{Psychopathie}

1. Ich mag es mich an Autoritäten zu rechen.

2. Ich meide gefährliche Situationen.

3. Rache muss schnell und kalt serviert werden.

4. Menschen sagen oft, dass ich außer Kontrolle sei.

5. Es stimmt schon, dass ich gemein zu anderen sein kann.

6. Menschen bereuen es immer, wenn sie sich mit mir anlegen.

7. Ich bin noch nie mit dem Gesetz in Konflikt gekommen.

8. Ich genieße es Sex mit Personen zu haben, die ich kaum kenne.

9. Ich würde alles sagen, um zu bekommen, was ich will.

Open Access This article is distributed under the terms of the Creative Commons Attribution 4.0 International License (http:// creativecommons.org/licenses/by/4.0/), which permits unrestricted use, distribution, and reproduction in any medium, provided you give appropriate credit to the original author(s) and the source, provide a link to the Creative Commons license, and indicate if changes were made.

\section{References}

Arbuckle, J. L. (1997). Amos users' guide version 3.6. Chicago: Small Waters.

Bollen, K. A., \& Long, J. S. (1993). Testing structural equation models. Newbury Park: Sage.

Bushman, B. J., \& Baumeister, R. F. (1998). Threatened egotism, narcissism, self-esteem, and direct and displaced aggression: Does selflove or self-hate lead to violence? Journal of Personality and Social Psychology, 75, 219-229. doi:10.1037/0022-3514.75.1.219.

Christie, R., \& Geis, F. (1970). Studies in Machiavellianism. New York: Academic.

Crede, M., Harms, P. D., Niehorster, S., \& Gaye-Valentine, A. (2012). An evaluation of the consequences of using short measures of the big five personality traits. Journal of Personality and Social Psychology, 102, 874-888. doi:10.1037/a0027403.

Emmons, R. A. (1987). Narcissism: Theory and measurement. Journal of Personality and Social Psychology, 52, 11-17. doi:10.1037/00223514.52.1.11.

Furnham, A., Richards, S. C., \& Paulhus, D. L. (2013). The dark triad of personality: A 10-year review. Social and Personality Compass, 7, 199-216. doi:10.1111/spc3.12018.

Furr, R. M., \& Bacharach, V. R. (2008). Psychometrics: An introduction. Thousand Oaks: Sage.
Goldberg, L. R., Johnson, J. A., Eber, H. W., Hogan, R., Ashton, M. C., Cloninger, C. R., \& Gough, H. C. (2006). The international personality item pool and the future of public-domain personality measures. Journal of Research in Personality, 40, 84-96. doi:10.1016/ j.jrp.2005.08.007.

Gosling, S. D., Rentfrow, P. J., \& Swann Jr., W. B. (2003). A very brief measure of the big five personality domains. Journal of Research in Personality, 37, 504-528. doi:10.1016/S0092-6566(03)00046-1.

Hambleton, R. K. (2001). The next generation of the ITC test translation and adaptation guidelines. European Journal of Psychological Assessment, 17(3), 164-172. doi:10.1027//1015-5759.17.3.164.

Hare, R. D., \& Neumann, C. S. (2008). Psychopathy as a clinical and empirical construct. Annual Review of Clinical Psychology, 4, 217 241. doi:10.1146/annurev.clinpsy.3.022806.091452.

Henning, H., \& Six, B. (2008). Machiavellismus. In A. Glöckner-Rist (Ed.), Zusammenstellung sozialwissenschaftlicher Items und Skalen. ZIS Version 12.00. Bonn: GESIS.

Jonason, P. K., \& Luévano, V. H. (2013). Walking the thin line between efficiency and accuracy: Validity and structural properties of the dirty dozen. Personality and Individual Differences, 55(1), 76-81. doi:10.1016/j.paid.2013.02.010.

Jonason, P. K., \& Webster, G. D. (2010). The dirty dozen: A concise measure of the dark triad. Psychological Assessment, 22, 420-432. doi:10.1037/a0019265.

Jonason, P. K., Li, N. P., Webster, G. W., \& Schmitt, D. P. (2009). The dark triad: Facilitating short-term mating in men. European Journal of Personality, 23, 5-18. doi:10.1002/per.698.

Jonason, P. K., Koenig, B. L., \& Tost, J. (2010). Living a fast life strategy: The dark triad and life history theory. Human Nature, 21, 428-442. doi:10.1007/s12110-010-9102-4.

Jones, D. N., \& Paulhus, D. L. (2010). Different provocations trigger aggression in narcissists and psychopaths. Social and Personality Psychology Science, 1, 12-18. doi:10.1177/1948550609347591.

Jones, D. N., \& Paulhus, D. L. (2014). Introducing the short dark triad (SD3): A brief measure of dark personality trait. Assessment, 21(1), 28-41. doi:10.1177/1073191113514105.

Küfner, C. P., Dufner, M., \& Back, M.D. (2014). Das Dreckige Dutzend und die Niederträchtigen Neun - Kurzskalen zur Erfassung von Narzissmus, Machiavellismus und Psychopathie. [the dirty dozen and the naughty nine - short scales for the assessment of narcissism, Machiavellianism and psychopathy]. Diagnostica, 1-16. doi:10. 1026/0012-1924/a000124.

Lee, K., \& Ashton, M. C. (2005). Psychopathy, Machiavellianism, and narcissism in the five-factor model and the HEXACO model of personality structure. Personality and Individual Differences, 38(7), 1571-1582. doi:10.1016/j.paid.2004.09.016.

Lee, K., Ashton, M. C., Wiltshire, J., Bourdage, J. S., Visser, B. A., \& Gallucci, A. (2013). Sex, power, and money: Prediction from the dark triad and honesty-humility. European Journal of Personality, 27, 169-184. doi:10.1002/per.1860.

Malesza, M., \& Ostaszewski, P. (2016a). Dark side of impulsivity Associations between the dark triad, self-report and behavioral measures of impulsivity. Personality and Individual Differences, 88, 197-201.

Malesza, M., \& Ostaszewski, P. (2016b). The utility of the dark triad model in the prediction of the self-reported and behavioral risktaking behaviors among adolescents. Personality and Individual Differences, 90, 7-11.

Maples, J. L., Lamkin, J., \& Miller, J. D. (2014). A test of two brief measures of the dark triad: The dirty dozen and short dark triad. Psychological Assessment, 26(1), 326-331. doi:10.1037/a0035084.

Miller, J. D., Few, L. R., Seibert, L. A., Watts, A., Zeichner, A., \& Lynam, D. R. (2012). An examination of the dirty dozen measure: A cautionary tale about the costs of brief measures. Psychological Assessment, 24, 1048-1053. doi:10.1037/a0028583.

Mulaik, S. (2007). There is a place for approximate fit in structural equation modelling. Personality and Individual Differences, 42, 883-891. 
Paulhus, D. L., \& Williams, K. M. (2002). The dark triad of personality: Narcissism, Machiavellianism, and psychopathy. Journal of Research in Personality, 36, 556-563. doi:10.1016/S00926566(02)00505-6.

von Collani, G. (2008). Modifizierte deutsche Versionen des Narcissistic Personality Inventory (NPI-d). In A. Glöckner-Rist (Ed.), Zusammenstellung sozialwissenschaftlicher Items und Skalen. ZIS Version 12.00. Bonn: GESIS.

von Collani, G., \& Herzberg, P. Y. (2003). Eine revidierte Fassung der deutschsprachigen Skala zum Selbstwertgefühl von Rosenberg. [A revision of the Rosenberg Self-Esteem Scale in German language].
Zeitschrift für Differentielle und Diagnostische Psychologie, 24, 37. doi:10.1024//0170-1789.24.1.3.

von Collani, G., \& Werner, R. (2005). Self-related and motivational constructs as determinants of aggression. An analysis and validation of a German version of the buss-Perry aggression questionnaire. Personality and Individual Differences, 38(7), 1631-1643. doi:10. 1016/j.paid.2004.09.027.

Williams, K.M., Nathanson, C., \& Paulhus, D.L. (2003). Structure and validity of the self-report psychopathy scale-III in normal populations. Toronto, Canada: Presentation at the 111 th annual convention of the American Psychological Association. 